\title{
Automatic Strain Gauge Balance Design Optimization Approach and Implementation Based on Integration of Software
}

\author{
Guangwei Xiang, Peng Mi, Guoqing Yi, Chao Wang, Wei Liu \\ China Aerodynamics Research \& Development Center, Mianyang Sichuan 621000, China, mipeng2016@126.com
}

\begin{abstract}
The traditional wind tunnel strain balance design cycle is a manual iterative process. With the experience and intuition of the designer, one solution that meets the design requirements can be selected among a small number of design solutions. This paper introduces a novel software integration-based automatic balance design optimization system (ABDOS) and its implementation by integrating professional design knowledge and experience, stepwise optimization strategy, CAD-CAE software, self-developed scripts and tools. The proposed two-step optimization strategy includes the analytical design process (ADP) and the finite element method design process (FEDP). The built-in optimization algorithm drives the design variables change and searches for the optimal structure combination meeting the design objectives. The client-server based network architecture enables local lightweight design input, task management, and result output. The highperformance server combines all design resources to perform all the solution calculations. The development of more than 10 balances that have been completed and a case study show that this method and platform significantly reduce the time for design evaluation and designanalysis-redesign cycles, assisting designers to comprehensively evaluate and improve the performance of the balance.
\end{abstract}

Keywords: Strain gauge balance, automatic design, structural optimization, software integration, optimization strategy.

\section{INTRODUCTION}

Strain gauge balances are widely employed in aerodynamic force measurement systems during wind tunnel tests. More than $70 \%$ of the tests utilize this kind of multicomponent force and moment measure instrumentations to estimate aerodynamic loads [1]. With today's increasing requirements of aerodynamic experiments for aerospace vehicles, ground vehicles, modern and unique architectures, etc., customers are concerned much more about the performance of the wind tunnel force measurements [2]-[5]. Therefore, wind tunnel balance developing technology has been focused and promoted for more than 100 years to achieve higher accuracy of steady and fluctuating force measurements [6]-[9].

The state-of-the-art design philosophy is the first and one of the most challenging areas of the balance development procedure which is an integration of all aspects of design, material selection, manufacture, thermal compensation, strain gauging and wiring, calibration, etc. [10], [11]. The calculation and optimization of the mechanical structural design determine most of the characteristics of the balance performance such as sensitivity, accuracy, safety, stiffness, and so on. Moreover, once the balance has been fabricated according to the original design, nothing can be changed during the use of the wind tunnel for decades, even if some structural issues have been found by the calibration. Consequently, improvements in wind tunnel balance design techniques are highlighted as the benchmark to consider advancements facilitated by researchers and facility requirements [12].

Early on, the strain gauge balance design including automated shape optimization [13] was primarily based on classical hand calculations or experiments until in the 1990s, the Finite Element Method (FEM) was exploited thoroughly in the structural optimization [14], [15]. Later, the design of experiments (DOE) methodology, the response surface methodology (RSM) or other optimization algorithms combined with FEM analysis were proposed to efficiently explore the design variables in the multidimensional design space in order to rely lightly on the experience and intuition of the balance designer [16]-[18]. Currently, most balance designers still prefer to obtain a balance by CAD and CAE tools, respectively [19]-[24], because of the discrete knowledge and computational support tools. However, these calculation and optimization systems based on commercial software or self-developed programs are often independent and not easy for personal or collaborative use. The loop of design-evaluate-redesign is always a complex and timeconsuming iteration. Furthermore, the design qualities of many balances depend heavily on the individual abilities because engineering designers choose the solution by manually adjusting design parameters. Thus, there is still a strong need of CAD-CAE tools and design experience integration for simplified, automatic, collaborative and optimum balance design. 
Today's computer-aided design techniques have many successful integrations of CAD-CAE and optimization tools to implement industrial product design systems [25]-[28]. These design applications were developed to facilitate the structural optimization in the different discipline fields with basically identical concepts and, therefore, designers have benefited more from them. For example, in the literature [25] a structural optimization common application was built by common scripting and programming languages to integrate commercial CAD-CAE tools with a presentation of a framework. The optimization process is well done automatically and seamlessly without user interaction. However, this meta-modeling technique and structural optimization method based on absolute simulation technology does not involve design experience and expertise, nor can it be directly applied to strain gauge balance design. In addition, each of the above optimization techniques typically has a series of applications that always depend on actual needs.

The strain gauge balance design optimization has its own characteristics and design philosophy [1], [10]. Therefore, the final structural design greatly accords to the balance design criterion and knowledge, which is in a conflict with the automated software integration concept. Although it was predicted that the use of FEM for routine balance design is not possible at the beginning of this century [10], the development of the knowledge-based integration system may change this [26]. Hence, a dedicated combination platform of the manually accumulated design knowledge and software tools will need to be developed to obtain a high-quality balance structure with a lower cost.

In order to exploit the advantages of both computer-aid tools and routine balance design knowledge, we developed and introduced an automatic balance design optimization system (ABDOS) that permits a seamless integration between commercial $\mathrm{CAD}$ and $\mathrm{CAE}$ tools in conjunction with engineering knowledge and applications. This method widens the existing enterprise knowledge portal (EKP) with the discrete design tools such as classic hand design (Excel), modeling tools (CATIA), FEM analysis (ANSYS), and optimization tool (iSIGHT). The unique strain gauge balance stepwise optimization strategy was proposed for knowledge integration. Moreover, this approach also eases the designer's labor force and enhances the collaborative engineering design by the friendly graphical user interface (GUI) wrapping and remote web service based EKP system upgrade.

\section{BALANCE OPTIMIZATION TECHNIQUES}

\section{A. Design principles}

Six-component conventional strain gauge balances are designed to obtain electrical signals generally exploiting the deformation of a definite mechanical structure for the conversion of mechanical signals either as a sting balance, a ring-type balance or a block balance. The combined geometry of the elastic sensing elements can be diverse according to the different design requirements. Thus, it is difficult to generate a uniform analytical formula for resolving all kinds of balances. However, the electrical signal vector is a function of forces (moments) and geometric parameters based on the elastic strain measurement principle. Therefore, for a common six-component strain gauge balance, the electrical signals for structural design can be expressed as follows:

$$
\left\{\begin{array}{l}
S_{1}=f\left(F_{X}, \boldsymbol{X}_{1}\right) \\
S_{2}=f\left(F_{Y}, \boldsymbol{X}_{2}\right) \\
S_{3}=f\left(F_{Z}, \boldsymbol{X}_{3}\right) \\
S_{4}=f\left(M_{X}, \boldsymbol{X}_{4}\right) \\
S_{5}=f\left(M_{Y}, \boldsymbol{X}_{5}\right) \\
S_{6}=f\left(M_{Z}, \boldsymbol{X}_{\mathbf{6}}\right)
\end{array}\right.
$$

where $\mathrm{S}_{1}$ to $\mathrm{S}_{6}$ represent the output signals of the balance components, $\boldsymbol{X}_{\boldsymbol{1}}$ to $\boldsymbol{X}_{\boldsymbol{6}}$ are the sets of geometric parameters (design variables) for the elastic sensing elements, $F_{X}, F_{Y}, F_{Z}$, $M_{X}, M_{Y}$, and $M_{Z}$ (Fig.1.) are the load components of the required measurement. Commonly, the load components include three forces and three moments to be measured on the model during the wind tunnel test.

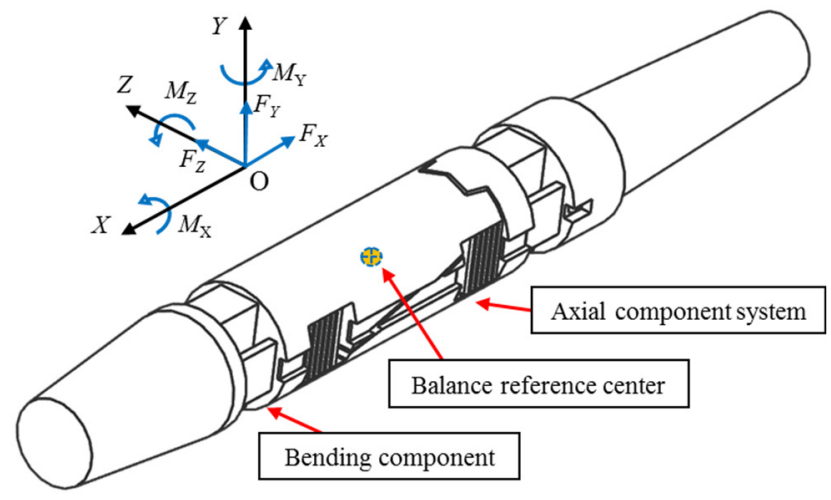

Fig.1. Balance axes system.

The basic working principle of a strain gauge balance (1) is a popular and easy method for approximated design. In a specific balance design task, the forces and the moments of the design loads are usually given by the customers. The signal of some components may be coupled with the others. For example, in a common block balance $S 2, S 4$, and $S 6$ are dependent upon each other although it can be decoupled through the post-data processing. Due to the test situation and limited design space, the sensitivity of each signal $S 1$ to $S 6$ is difficult to optimize simultaneously within a reasonable range. However, it should be as close as possible to a certain target. In fact, the unknown design variables $\boldsymbol{X}_{\boldsymbol{1}}$ to $\boldsymbol{X}_{\boldsymbol{6}}$ of each component are the design output. To determine the appropriate variables $\boldsymbol{X}_{\boldsymbol{1}}$ to $\boldsymbol{X}_{\boldsymbol{6}}$, it is impossible to solve (1) directly because there are multiple structures that can meet the same design requirement.

It is clear that the feasible designs can be estimated in advance based on the principles of mechanics while the design load and parameters of the elastic sensing elements are already known. The optimal design can be selected by comparison of the feasible designs. Furthermore, the design equations included in (1) can be assorted according to the 
distinct measurement principles. Table 1. shows these formulas and some basic calculation items. Here $E, G, F, M$, $v, A, W, W_{p}$, and $K$ are Young's modulus of elasticity, shear modulus, force, moment, Poisson ratio, cross section area, section modulus of bending, section modulus of torsion, and stiffness, respectively. With a given geometry and the material properties, the predictable stress, strain, deformation, and output signal can be calculated, respectively [1], but not the other way around.

The common elastic sensing elements are based on the mechanics theory of tension (compression), bending, and twisting. The analytical design process (ADP) is performed using the design equations listed in Table 1 . In order to obtain reasonable electrical signals, sufficient strain should be generated on the areas where the strain gauge is located. The strain can be calculated with the stress by Hooke's law. The stress is determined by the geometric and material properties of the structure. Meanwhile, the structural deformation under the force or moment can be also obtained to evaluate the stiffness of the structure. Correspondingly, the finite element design process (FEDP) is just another way to solve these design equations in implicit forms with the ADP idea mentioned above. Therefore, the balance design is essentially a process of mechanical analysis.

In addition, the role of individual abilities and experience in balance design cannot be ignored at any time. In the process of design techniques evolving and practical problem solving, some interesting facts were discovered. 1) Commonly used sensing elements are enumerable. After years of balance development, these various elements have been employed to form the conventional strain balances. For instance, the sting balances usually exploit bending components (e.g., single beam or multi-column beams) to measure forces $F_{Y}, F_{Z}$ and moments $M_{Y}, M_{Z}$ as well as $M_{X}$ with either bending or twisting. The appropriate structure is selected according to the designer's subjective judgment. 2) The design equations for common structures are already available and validated. Although the ADP has some assumptions and significant calculation errors, designers for sensitivity evaluation in the preliminary design phase normally accept it. Accurate evaluations, such as stress and deformation predictions, primarily depend on the FEDP. 3) A large number of design cases have provided a down-to-earth practical basis for automatic design optimization. A new design is usually the optimization result of some existing balances because there are not too many innovations in sensing elements themselves. 4) Sensitivities are not suitable as design objective functions and the maximum stiffness is often considered as an optimization target. Since it is much easier to solve a single objective optimization problem than a multi-objective one, it is sensible to constrain the sensitivity of the output signal to a reasonable range for each component, respectively.

Table 1. Principle design equations.

\begin{tabular}{|c|c|c|c|c|c|}
\hline Sensor type & Geometry/ Material & Stress $(\sigma, \tau)$ & Strain $(\varepsilon, \gamma)$ & Deformation $(\delta, \varphi)$ & Signal $(\mathrm{S})$ \\
\hline $\begin{array}{c}\text { Tension } \\
\text { Compression }\end{array}$ & $\begin{array}{c}A=f(\boldsymbol{X}) \\
K=f(E, \boldsymbol{X})\end{array}$ & $\begin{array}{c}\sigma_{\text {tension }}=F / A \\
\sigma_{\text {comp }}=v F / A\end{array}$ & $\varepsilon=\sigma / E$ & $\delta=F / K$ & $\boldsymbol{S}=f(\varepsilon)$ \\
\hline Bending & $\begin{array}{c}W=f(E, \boldsymbol{X}) \\
K=f(E, \boldsymbol{X})\end{array}$ & $\sigma=M / W$ & $\varepsilon=\sigma / E$ & $\delta^{\prime \prime}=M(x) / K$ & $\boldsymbol{S}=f(\varepsilon)$ \\
\hline Twisting & $\begin{array}{c}W_{p}=f(G, \boldsymbol{X}) \\
K=f(G, \boldsymbol{X})\end{array}$ & $\tau_{\max }=M / W_{p}$ & $\gamma=\tau / G$ & $\varphi=M / K$ & $\boldsymbol{S}=f(\gamma)$ \\
\hline
\end{tabular}

\section{B. Mathematical model}

The multi-component balance optimization problem can be uniformly described as:

$$
\min (1 / K(\boldsymbol{X}))
$$

Subjected to:

$$
\begin{gathered}
f^{\text {ineq }}(\boldsymbol{X}) \leq 0 \\
f^{e q}(\boldsymbol{X})=0 \\
\boldsymbol{X}_{\text {min }} \leq \boldsymbol{X} \leq \boldsymbol{X}_{\text {max }} \\
\boldsymbol{S}_{\text {min }} \leq \boldsymbol{S} \leq \boldsymbol{S}_{\text {max }}
\end{gathered}
$$

where $1 / K(\boldsymbol{X})$ is the objective function, $f^{\text {ineq }}(\boldsymbol{X})$ and $f^{e q}(\boldsymbol{X})$ are inequality and equality constraints, respectively, $\boldsymbol{X}_{\min }$ and $\boldsymbol{X}_{\max }$ are the lower and upper limits of the design variable vectors, respectively, $S_{\min }$ and $S_{\max }$ are the lower and upper limits of the signal vectors, respectively.
Minimizing the reciprocal of stiffness summation $K(\boldsymbol{X})$ is the objective function as expressed in (2). The stiffness of some simple structures, such as cantilever beams with specific dimensions, can be calculated by the formulas and methods in Table 1. However, in structures with complex geometric features accurate results by ADP are difficult to obtain. Equivalent to this objective function is the structural deformation $\delta=f(\boldsymbol{X})$, which is commonly used in FEDP. It is essentially the same but more intuitive and convenient for designers, because it is easy to extract the deformation somewhere in any FEM software.

In $(3), f^{\text {tneq }}(\boldsymbol{X})$ is a set of inequality constraints. For example, the maximum stress on the structure should be less than the allowed stress, and the interference between the components should be less than a certain threshold. Stress safety and interference specifications are design factors that must be concurrently taken into account in any balance design optimization. Stress concentration and interference should be minimized to some suitable value, respectively. These technical specifications are difficult to predict by ADP, but it has become a routine means by FEDP. 
In (4), $f^{e q}(\boldsymbol{X})$ is a set of equality constraints, including some given dimensions (constants), or a set of determined discrete variables. For a sting balance, the number of support flexures in the axial component system can be a multiple of four. Generally, it would be 12, 16, 20, 24, 28, and 32. Some dimensions that do not need to be treated as variables can also be considered as equality constraints, such as the diameter of the balance and the dimensions of the interface.

The design variables $\boldsymbol{X}$ in (5) are constrained by the design space according to the balance structures. In general, all design variables for a 6-component balance can reach dozens or more. Taking into account processing and optimization efficiencies, these values should be sensitively analyzed by DOE and preferably rounded rather than using random arbitrary values.

Equation (6) is the constraints for output signal sensitivity vector S. A set of boundaries S1 to S6 are given according to the design requirements, respectively.

The unified mathematical model can be used in both ADP and FEDP to optimize a conventional strain gauge balance. In the optimization based on FEDP, more objective functions can be extended to form a multi-objective optimization, such as minimum interference or minimum heat output of the axial component of a sting balance, which will make the balance optimization more complicated. The stiffness objective function provides a criterion for evaluating the design quality instead of the designer's intuition. The goal of the ABDOS is to automatically find a set of $\boldsymbol{X}$ in the entire design space so that the structure satisfies all the constraints and the objective function is minimized instead of carrying out the optimization process by the human brain.

\section{Automatic optimization strategy}

A great deal of existent knowledge and experience of balance design accumulated for a long time shows that both ADP and FEDP have their own advantages and disadvantages. ADP is an approximating calculation approach that has low computing cost with acceptable accuracy in solving stress and stiffness. For example, when calculating the shear stress of multi-column beams, the deviation may exceed $20 \%$. But its great advantage is that the parameters can be easily adjusted to get new design results immediately. FEDP can be free of structural complexity and the simulation result is more accurate. In some cases, FEDP can help designers obtain credible results (e.g., stress concentration, deformation) which cannot be possibly obtained by ADP. However, FEDP is limited by the computing performance and the scale of the simulation. Also, it is more time consuming and requires higher individual abilities for designers. Therefore, ADP is suitable for finding the approximate solutions or possibilities in the preliminary design phase to meet the sensitivity requirements, and FEDP is more appropriate for the final optimization design.

In order to use the advantages of ADP and FEDP, an automatic optimization strategy for balance development is proposed. This strategy includes sensor-based modeling methods, stepwise optimization approaches, and optimization algorithms-driven design updates, as shown in Fig.2.

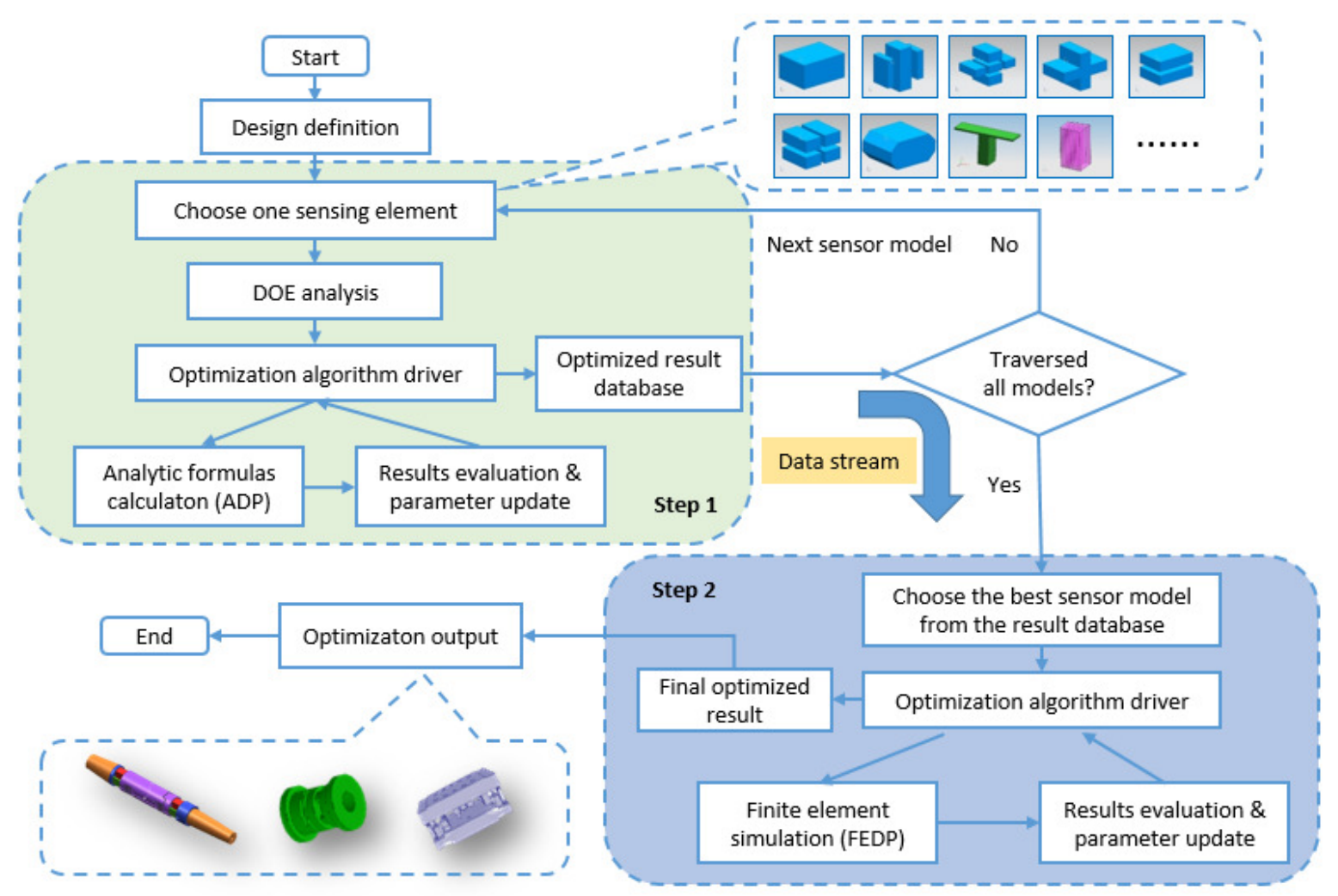

Fig.2. Strain gauge balance design optimization strategy. 
Parametric sensing elements models are established by mathematical and geometric methods, respectively. As discussed earlier, although the balances with a combination of elastic sensing elements are numerous, basically there are only a few dozen sensitive structures (e.g., column beams, Tshaped axial component, $\pi$-shaped axial component, etc.). Whether the balance is assembled or monolithic, it can be split into basic sensing elements for design consideration individually. These basic sensing elements can be defined, parameterized and documented in files or scripts in advance of both ADP and FEDP for data exchange and automatic updating.

The well-documented models are one-to-one correspondence built in a component library with identical parameters definition. 3D modeling software (e.g., CATIA) can also call these files to generate solid models for result visualization and manufacture eventually. This component library can also be continuously expanded and maintained for reuse. This is the important advantage of modeling when the optimization processes require many iterations of ADP and FEDP evaluations. Although it is cumbersome to build this library, the existing design resources have a good foundation and only minor modifications are needed. The conventional balances include about two dozens of sensing element models, which can be combined as different types of sting balances, ring balances, and block balances.

The stepwise optimization process involves two steps: 1) Combining sensor models from the component library and find the global sequential feasible schemes by feasibility, 2) Further dimension exploring and validating based on the result of step one to obtain the final optimum structure. This process has a significant advantage of being balanced in both time and quality because of the combination of ADP and FEDP.

The first step of optimization is ADP optimization. The main task is to find the best combination for design conditions from the component library and establish the basic structure of the balance. The second step is FEDP optimization, which further optimizes and evaluates the first-step preferred results to obtain an optimal design that can be used in practical engineering.

The main task of step 1 is to find the best combination for design conditions and establish the basic structure of the balance. With the fast speed of ADP, a best basic design solution can be searched from hundreds of combinations driven by the optimization algorithms in an acceptable time. The design of experiment analysis can clarify which variables have a significant impact on the results to reduce the needed time. This initial exploratory optimization is not very accurate, but it is sufficient as a preliminary design for structure selection.

The result of step 1 is confirmed manually so that the design flexibility is still in the hands of the designer. According to the optimization objective of (2), the most feasible combination scheme can be selected from the result database as the starting point for detailed optimization. More than one scheme can be further optimized, respectively. The FEM model further refined with detailed geometric features (e.g., chamfers) will be directly selected by the designer as design variables for FEDP optimization. The definite geometry customizing simulations are supported by a powerful computing server so that more accurate stress, deformation, interference, and even thermal analysis results are possible to be evaluated by this process in an acceptable time.

Both steps are achieved through the mature optimization algorithm to obtain the solutions, which greatly reduces the difficulty of the system implementation. Many optimization softwares integrate these algorithms without any programming. These algorithms can directly drive design updates until feasible solutions are found and sorted.

Considering the specific implementation of the design optimization strategy, a data flow based programming is applied to seamlessly integrate ADP and FEDP. Both processes share a common set of parameters defined for data exchange and updates. Some necessary modularized software components are developed to wrap all the existent CAD-CAE tools based on the EKP network platform to achieve remote and automatic optimization at the enterprise level.

\section{AUTOMATIC MODELING AND OPTIMIZATION INTEGRATION}

\section{A. Overall framework of optimization tools}

The proposed architecture of the ABDOS is based on software integration and a developed server tool (Fig.3.). It is probably divided into two parts, the local user interface and the remote service support. This strategy includes sensorbased modeling methods, stepwise optimization approaches and optimization algorithms driven design updating, and remote service support. A few front-end GUI client applications on local PC and a back-end EKP task management tool on the server are programmed to access the ABDOS services over the company-internal network in order to meet the distributed designs in the different locations. The management tool controls all the optimization processes and data translations. Each optimization process can be viewed as a ready-made plug-in which is wrapped in some commercial CAD-CAE tools (e.g., CATIA, ANSYS) and engineering programs (e.g., iSIGHT, Excel). All modeling processes, calculations, simulations and parameter searches are running on the remote server. Another dedicated data server is equipped to manage the task and data.

Due to the existing balance development resources, software tools including Excel, ANSYS, CATIA, and iSIGHT have evolved into providing design optimization solutions. The DOE analysis and automatic optimization are carried out through the integration of CATIA, ANSYS, and Excel software in iSIGHT. As a powerful optimization tool, iSIGHT is professional for integrating CAD-CAE tools, exploring optimal results and automating workflow. iSIGHT also provides comprehensive optimization algorithms, such as Hooke-Jeeves direct search, sequence quadratic programming, non-dominated sorting genetic algorithm II and pointer automatic optimizer that automatically evaluates optimization results, which are suitable and effectively adapted to different situations for the automatic strain balance optimization problem [29]. These benefits will greatly reduce design costs and improve the quality and efficiency of the balance development so that the closed-loop automatic optimization and remote design functions can be realized. 


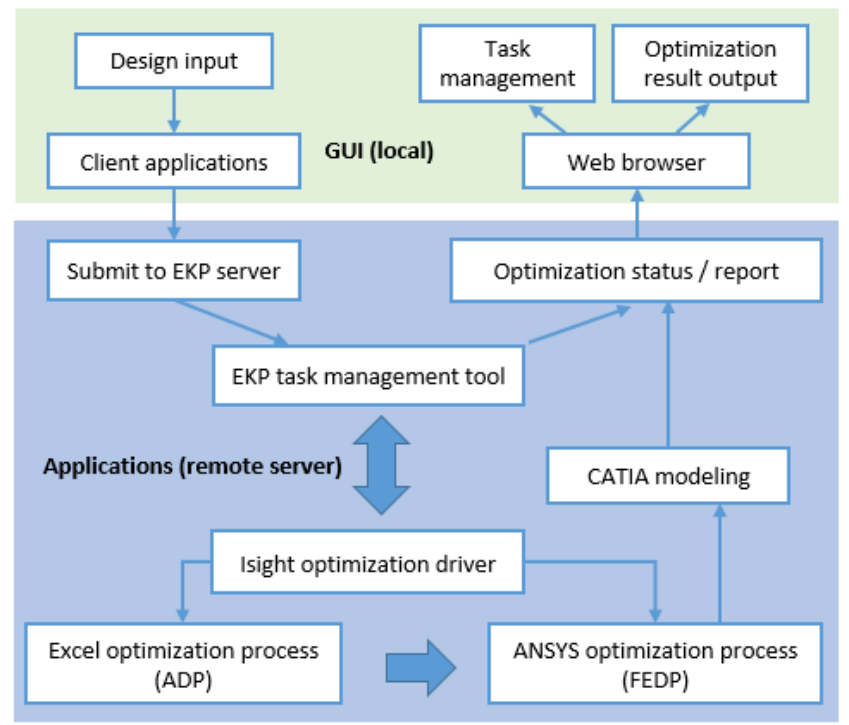

Fig.3. Overall framework.

The self-developed EKP task management tool configures the server correctly and associates the client software. It encapsulates the two types of optimization processes based on iSIGHT. Also, it is responsible for the sharing of all optimization processes, the local setting of balance structure parameters, task management, server resource allocation, and design result output. Users do not need to have an in-depth understanding of iSIGHT instead of a certain basic knowledge for the balance design. All the parameters that can be set by the user are directly selected and defined through the client applications. These parameters are mapped to the applications such as Excel, ANSYS, or CATIA so that designers can really pay attention to the balance design itself without spending a lot of effort on the learning and application of engineering software.

Excel is employed in the integration of ADP because this common office software makes it easy to generate formula calculation files and iSIGHT can easily drive these files with algorithms. Another reason is that these balance calculation sheets have been adopted by most balance engineers. They can be easily modified and improved to form an ADP standard library.

ANSYS is used for FEDP optimization with its classic mechanical calculation modules instead of workbench because it offers the means to automate the modeling, solution, and result extraction by the use of ANSYS parametric design language (APDL) programmed files. A FEDP standard library is built like the ADP library. These text files from the libraries can be easily parsed, mapped, and driven by iSIGHT.

\section{B. Automatic methodology}

iSIGHT drives Excel and ANSYS optimization processes independently in two different design phases. Fig.4. shows an example of the Excel optimization loop for the sting balance design. Components Input_Param and Result_Output are data exchange modules for storing and converting design input and result output. Component Input_Param also receives the user settings from the EKP task management tool to control the operation of the optimization component, including the selection of constraints and optimization algorithms. The design variables of the balance are directly managed and driven by the optimization component through parameters mapping. The optimization component is responsible for completing the search and parameter updates to obtain the best balance configuration. In this example, there are two Excel calculation files, Calc 5COM and Calc_XCOM, which are used for the ADPs of the bending component and axial component of the sting balance, respectively.

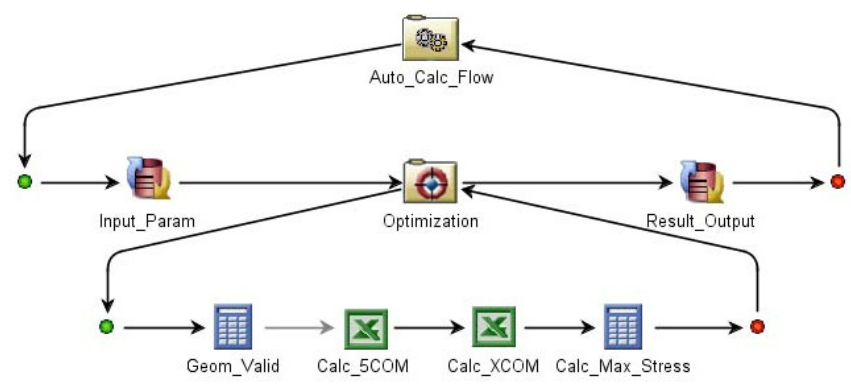

Fig.4. iSIGHT driving Excel optimization process.

Once such a process is created and verified, it can be reused. The ADPs for different sensing components can be realized by replacing different Excel calculation files. These files in the library built in advance can be configured with the corresponding parameters mapping (Fig.5.). These configurations should be debugged once to make sure the results are confident compared with FEM and saved as template files for recalls.

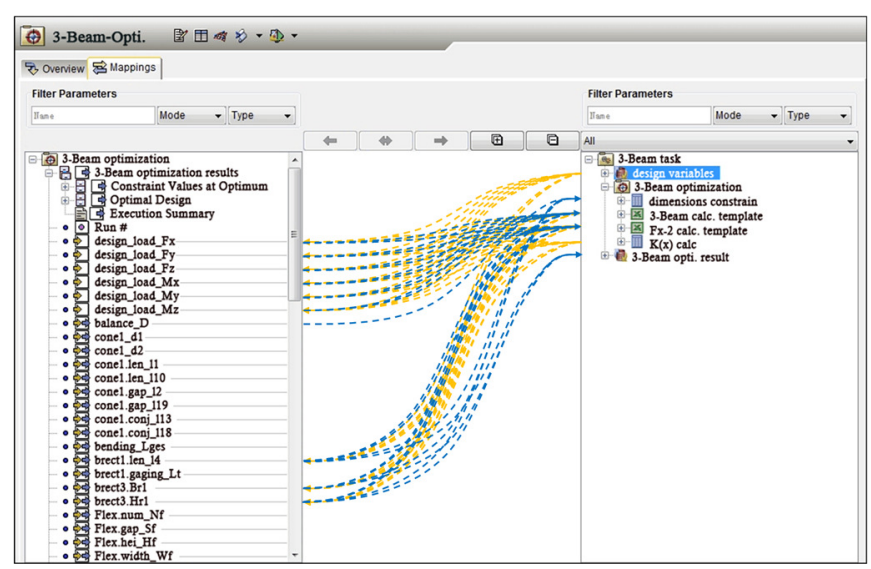

Fig.5. Design parameters mapping.

As Fig.5. shows, the parameters are mapping which is actually the process of reading the result from the calculation and writing the new parameters to the same Excel file driven directly by the optimization component. In this example, a three-beam sensing element with approximately a few dozen design variables is manipulated. The optimization component generates hundreds of sets of design parameters according to the optimization algorithm. These parameters are sequentially written into the corresponding cells of the Excel calculation 
file, then the optimization component reads the calculation results immediately and stores them in the results database. After all the parameter combinations have been calculated, the optimal solutions that satisfy the constraints are sorted by feasibility according to the defined objective as the design output of the preliminary design.

Fig.6. shows an example of a typical ANSYS optimization process that can be considered as an upgraded version of the Excel optimization process with a more complicated configuration because of the detailed calculations. Compared to the ADP optimization, the Excel calculation files are replaced by the component Bal_Mech_Sim which is the APDL-based FEM simulation command stream file mentioned previously. Like the ADP optimization, the FEDP optimization also needs to map all the parameters once and the simulation is verified by a manual FEM run. An additional component Final_Recalc of further refining simulation for the verification is executed once after the optimization is finished. For the sting balance optimization, more simulations and items are included. In this example, the heat transfer calculations are added to evaluate the temperature gradient because the user has previously defined it as a multi-objective problem. The component Bal_Model will call CATIA for 3D modeling based on the final optimal structure to visualize the optimization results. Unlike general FEM simulations,
CATIA does not create any model for the calculation because the APDL command stream is fully capable of building any parametric models.

The APDL command stream file is the key to automating the FEM simulation analysis. The text script file includes all simulation processes such as modeling, meshing, solving, result extracting, etc., which can be mapped by iSIGHT as well as Excel files. Although these APDL files usually include about 1000 to 2000 lines of commands, it is possible to automate the recording of these scripts by ANSYS GUI operation, making them reusable. All calculation results are saved to the ANSYS database, which can be reviewed according to user requirements. Also, a calculation report is generated for the optimization result display.

With the above-mentioned approaches, there are dozens of Excel optimization processes and ANSYS optimization processes based on the established iSIGHT. They are executable files in a standard template library manipulated by the EKP task management tool. These template files stored on the server can be upgraded to a new version or modified to other standard processes and released for users. Also, they can be downloaded to the local PC for viewing and modification. These optimization plug-ins are not only the integration of computational optimization tools but also the accumulation of design experience.

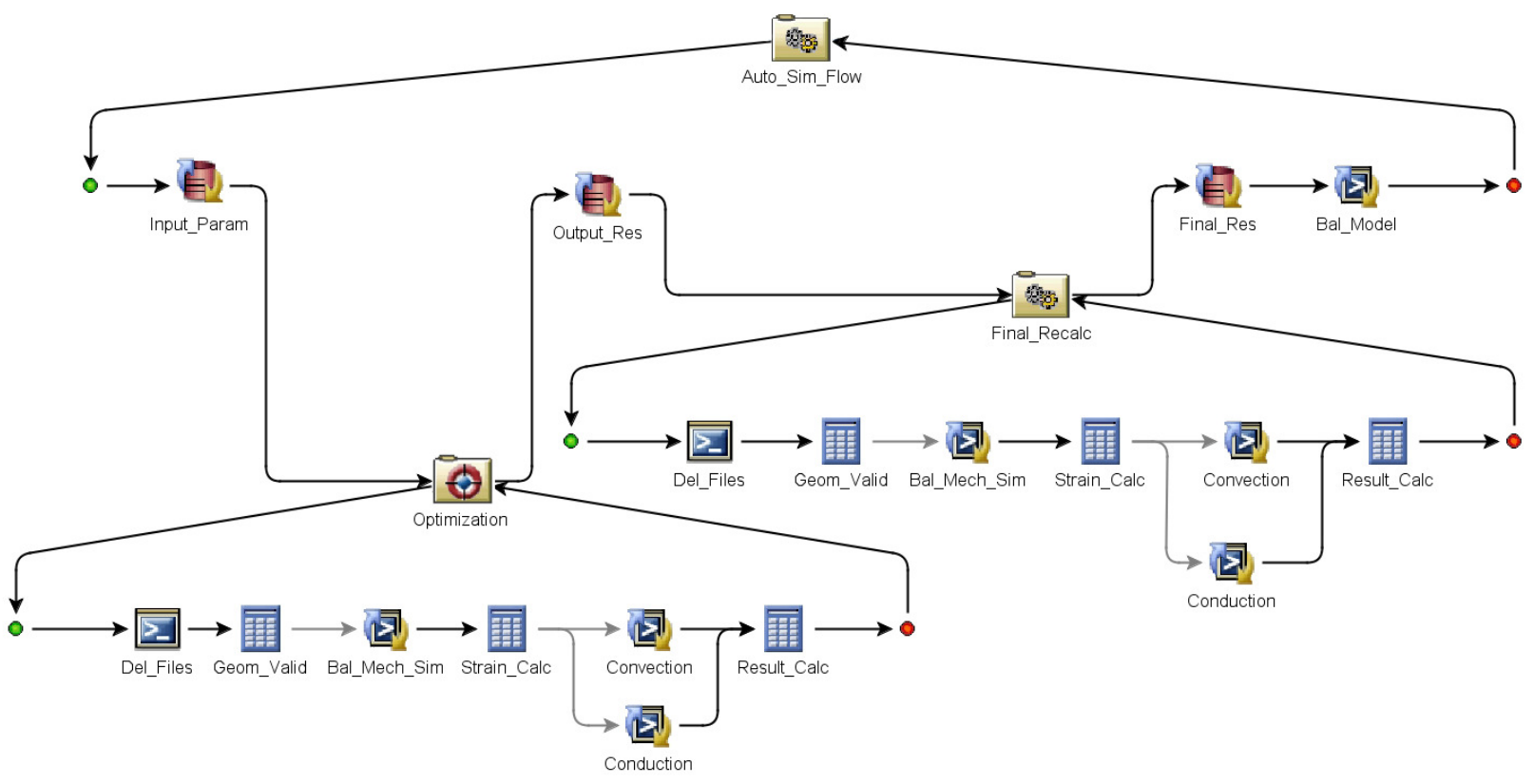

Fig.6. iSIGHT driving ANSYS optimization process.

\section{Integration and packaging}

At the time of this writing, ABDOS integrates 94 design optimization processes inherited and derived from 6 basic classes, respectively, as shown in Table 2 . Among them, more than $2 / 3$ of the optimization packages are for sting balances. Up to 42 variations of sting balance configurations can be generated by ABDOS for a conventional wind tunnel test. For the ring-type and block balances, there are 29 and 2 balance optimization processes, respectively. These processes have been carefully debugged and verified to ensure both the performance and the efficiency.

The typical user interfaces GUI and web services in the browser are shown in Fig.7. Any member of the balance design team is able to access ABDOS after going through the following 4 steps: client applications download (if not), design definition, optimization definition, task generation and confirmation of results. Usually, the Excel application is taken at first to search for some ideas, then the ANSYS application can be carried out for the final design. 
Table 2. Optimization packages for routine balances.

\begin{tabular}{|c|l|l|l|}
\hline No. & \multicolumn{1}{|c|}{ Client applications } & \multicolumn{1}{c|}{ Sensor Geometry } & \multicolumn{1}{c|}{ Optimization templates } \\
\hline 1 & Excel package for sting balances & $\begin{array}{l}7 \text { bending components } \\
3 \text { axial components }\end{array}$ & 21 Excel loops \\
\hline 2 & ANSYS package for sting balances & $\begin{array}{l}7 \text { bending components } \\
3 \text { axial components } \\
2 \text { kinds of slots }\end{array}$ & 42 ANSYS loops \\
\hline 3 & Excel package for ring-type balances & 13 configurations & 13 Excel loops \\
\hline 4 & ANSYS package for ring-type balances & 16 configurations & 16 ANSYS loops \\
\hline 5 & Excel package for block balances & 1 configuration & 1 Excel loop \\
\hline 6 & ANSYS package for block balances & 1 configuration & 1 ANSYS loop \\
\hline
\end{tabular}

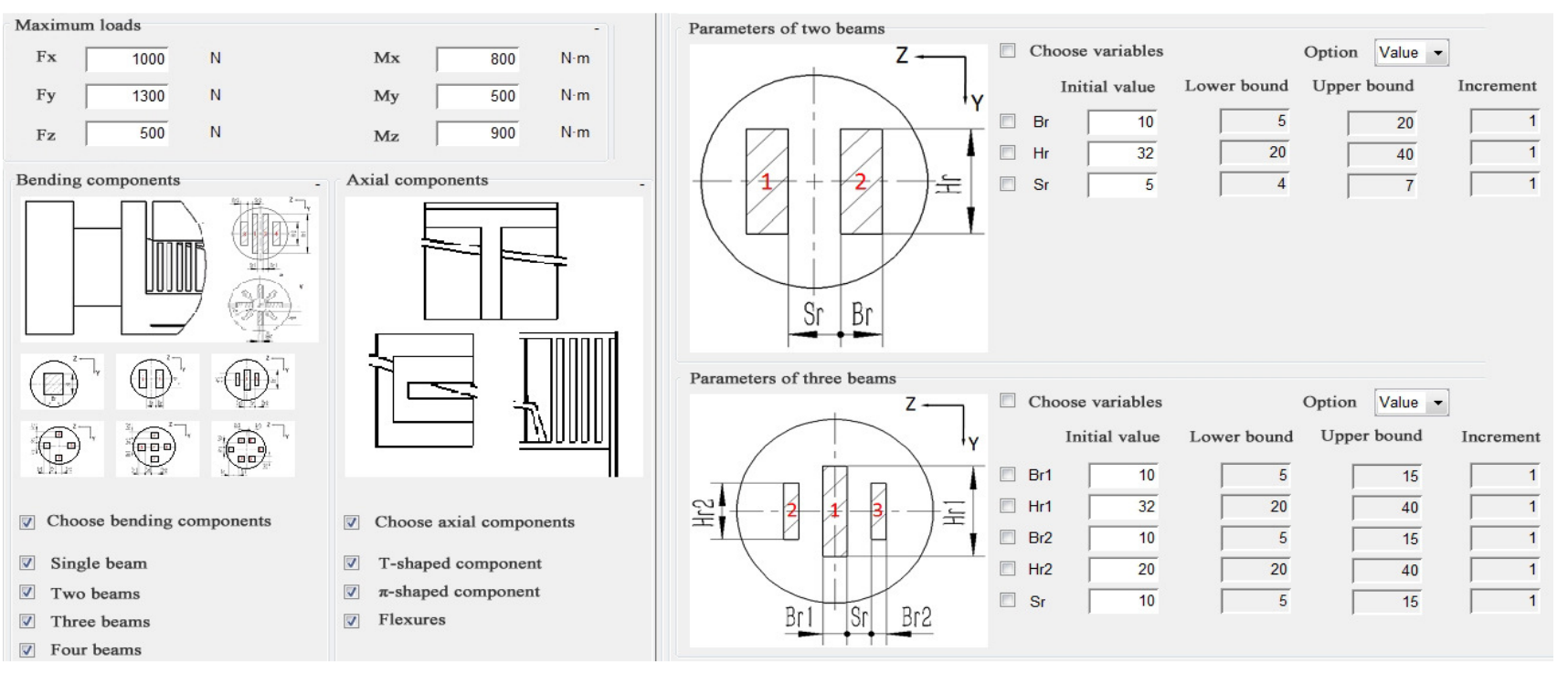

Fig.7. GUI of the client application for design input.

The design input interfaces of all the above optimization processes are packaged in 6 lightweight client applications developed by Java, which can be downloaded from the EKP webpage at the first time. This refers to launching a new design task or opening an existing task file.

The design definition consists of the balance load range setting, sensing elements selection, and design variables setting. At least one group of elements should be selected. Dimensions of all selected elements should be defined as variables or constant values. Variables should be initialized with the proper lower, upper limits and increment. The increment is set to reduce the computing cost and fit to the manufacture dimensions. For the ANSYS optimization application, the Excel parameters can be imported to the design definition as a start point. Appropriately labeled marks, tips and schematic drawings aid the user input the correct number of design variables and constraints.

During the optimization definition, the optimization problem is further determined, involving objective functions, sensitivity constraints, etc. The Excel optimization only has one objective function defined by (2) but there are more choices for ANSYS optimization (e.g., deformation, interference, thermal output minimizing, etc.).The task is subsequently saved to the database in ABDOS format for future retrieval, modifications, or executions.

The task generation is done with one mouse click to submit the task to the remote server. The tasks generated by designers distributed in different locations are put into the task queue that is managed by the upgraded EKP, which can be reviewed by logging on to the server through a web browser.

All the tasks and results can be retrieved from the database in the final step. All data files (inputs and results) can be saved to the database for future reference or analysis. The available results, such as 3D solid model, iSIGHT project files and the task report, can be downloaded from the summary webpage.

\section{CASE STUDY \\ A. Review of recent finished tasks}

The web service based and optimization algorithm driven ABDOS was put into application in 2016 providing an enhanced approach for conventional balance development. Through the software tools and design knowledge integration with sharing hardware resources, the balance design optimization ability has been significantly improved. 
ABDOS enables not only new designs or optimizations but also the verification of existing designs or optimizations based on existing balances. The 24-hour online server has been upgraded for running multiple tasks simultaneously. In the past two years, more than 10 balances have been successfully designed and optimized with a total of 200,000 times of the design-evaluate-redesign cycles being completed automatically. Most of these completed balance designs have been machined, calibrated, and tested successfully for different wind tunnels.

Table 3. shows the approximate time consumption of some representative projects. In fact, it is difficult to calculate the precise design optimization time of a project because it depends upon not only the design platform but also the capabilities of the designer. A specific task may need to run multiple times with different settings depending on the design difficulty and the designer's understanding of the task. It is clear that the average optimization time of a conventional balance design is reduced from ten days or dozens of days to several days compared to the traditional manual calculations.

The analysis time for a certain sensing element structure is in an acceptable range in both ADP and FEDP optimization. The Excel optimization process can achieve optimal results after approximately 0.28 hours for one element of optimum dimension searching. After an ANSYS optimization process task is submitted, it takes about 10.8 hours to get the optimization result. This is the advantage of the optimization strategy and design resource integration based on the highperformance computing server. However, the balance designers should take some time to understand the design task or have some attempts previously before the formal running. In general, multiple optimizations for one task are required to achieve satisfactory results because some parameters, such as the definition of the optimization problem, the boundary conditions of the design space, may need to be changed.

For a certain set of optimization definitions and design constraints, ABDOS can find several feasible sorted designs from hundreds or thousands of design schemes. The global optimal solution can be obtained compared with the traditional manual optimization based on the design experience and personal preference. Additionally, ABDOS reduces the difficulty for users to know a variety of CADCAE tools and engineering software. It is no longer necessary to be proficient in the technical optimization details instead of design input and result output by this seamless integration in the EKP platform, which is a kind of black-box design approach with strong engineering practicability.

Table 3. Some balance tasks accomplished by the design optimization system.

\begin{tabular}{|c|l|l|l|l|c|}
\hline No. & Designation & Completion & \multicolumn{1}{|c|}{ Balance type } & Application wind tunnel (WT) & $\begin{array}{c}\text { Time } \\
\text { consumed (h) }\end{array}$ \\
\hline 1 & $64 \mathrm{D}$ & Mar. 2016 & 6-component sting & $2.4 \mathrm{~m} \times 2.4 \mathrm{~m}$ Transonic WT & 66.5 \\
\hline 2 & $47 \mathrm{E}$ & Apr. 2016 & 6-component sting & $1.2 \mathrm{~m} \times 1.2 \mathrm{~m}$ Transonic \& Supersonic WT & 97.8 \\
\hline 3 & $24 \mathrm{G}$ & Aug. 2016 & 6-component sting & $0.6 \mathrm{~m} \times 0.6 \mathrm{~m}$ Transonic \& Supersonic WT & 80.1 \\
\hline 4 & TG02 & Jun. 2017 & 6-component block & $8 \mathrm{~m} \times 6 \mathrm{~m}$ Low Speed WT & 135.2 \\
\hline 5 & 50C & Nov. 2017 & 6-component ring-type & $\Phi 1 \mathrm{~m}$ Hypersonic WT & 88.9 \\
\hline 6 & $120 \mathrm{~B}$ & Oct. 2017 & 6-component ring-type & $2 \mathrm{~m} \times 2 \mathrm{~m}$ Supersonic WT & 121.3 \\
\hline 7 & $82 \mathrm{~A}$ & Jan. 2018 & 6-component sting & $2 \mathrm{~m} \times 2 \mathrm{~m}$ Supersonic WT & 103.9 \\
\hline
\end{tabular}

\section{B. Sting balance design and optimization}

The sting balance is widely used in high-speed wind tunnel tests and has a complex structure with distinct features, which is representative for the application of ABDOS. The following text will take a sting balance as an example to illustrate the application of the design optimization platform. An existing balance (balance A) employed in $2.4 \mathrm{~m} \times 2.4 \mathrm{~m}$ transonic wind tunnel is selected as a benchmark in order to verify the platform. Balance A exploits a simple rectangular beam as a bending component measuring forces and moments except the axial force, and a T-shaped beam to measure the axial force. The new design balance $\mathrm{B}$ with a diameter of $64 \mathrm{~mm}$ is the same as balance A. Table 4. shows the maximum loads of the two balances.

The design space is constrained to a cylinder with a diameter of $64 \mathrm{~mm}$ and a length of $180 \mathrm{~mm}$ excluding the interface that is fixed. The stress limit is $1240 \mathrm{Mpa}$ with a safety factor of 1.5. The minimum value of the sensitivity constraint is $S_{\min }=0.5 \mathrm{mV} / \mathrm{V}$, and the maximum value is set to $\mathrm{S}_{\max }=1.5 \mathrm{mV} / \mathrm{V}$ except for the axial component that is set to $1 \mathrm{mV} / \mathrm{V}$ according to the design criterion. It is desirable to get a new balance with better stiffness and minimal interferences.

Table 4. The maximum loads of balance A \& B.

\begin{tabular}{|c|c|c|c|c|c|}
\hline$F_{X}(\mathrm{~N})$ & $F_{Y}(\mathrm{~N})$ & $F_{Z}(\mathrm{~N})$ & $M_{X}(\mathrm{~N} \cdot \mathrm{m})$ & $M_{Y}(\mathrm{~N} \cdot \mathrm{m})$ & $M_{Z}(\mathrm{~N} \cdot \mathrm{m})$ \\
\hline 1400 & 15000 & 6000 & 600 & 700 & 1300 \\
\hline
\end{tabular}

According to the optimization strategy described in section 2, the Excel optimization process is used for the investigation of the initial structural schemes. All of the sensing elements for the sting balance are chosen and hence the boundaries of the geometric parameters are set to find some feasible combinations. Some feasible solutions were searched from 13839 designs, and the top five optimal structural combinations are listed in Table 5. 
Table 5. Sensitivities of optimum balance configurations.

\begin{tabular}{|c|c|c|c|c|c|c|c|c|c|}
\hline No. & $\begin{array}{c}\text { Bending } \\
\text { components }\end{array}$ & $\begin{array}{c}\text { Axial } \\
\text { components }\end{array}$ & $S_{1}$ & $S_{2}$ & $S_{3}$ & $S_{4}$ & $S_{5}$ & $S_{6}$ & $\begin{array}{c}1 / K(X) \\
\left(\times 10^{-6} \mathrm{~N}^{-1} \mathrm{~m}^{-2}\right)\end{array}$ \\
\hline 1 & 3 beams & T-shaped (A) & 0.65 & 0.90 & 0.80 & 0.78 & 0.94 & 1.12 & 5.07 \\
\hline 2 & 2 beams & $\pi$-shaped & 0.71 & 0.93 & 0.85 & 0.99 & 1.02 & 1.25 & 5.28 \\
\hline 3 & 1 beam (A) & $\pi$-shaped & 0.71 & 0.87 & 0.65 & 0.55 & 0.93 & 0.93 & 5.45 \\
\hline 4 & 4 beams & T-shaped & 0.65 & 0.95 & 0.77 & 0.93 & 1.05 & 1.16 & 5.78 \\
\hline 5 & 5 beams & $\pi$-shaped & 0.71 & 1.05 & 0.88 & 1.05 & 1.11 & 1.28 & 5.98 \\
\hline
\end{tabular}

These preliminary design results show that the bending component measurement can be realized by using different beams and the axial component can be a T-shaped beam or a $\pi$-shaped beam. Balance A is not the best configuration, the sensitivity of the rolling moment and the axial component can be improved. For balance B, several of these previous schemes can be chosen to be optimized in the next design phase. It is clear that the structural configuration of the 6component balance is not unique, but each feasible scheme has its own advantages and disadvantages. The designer needs to make a decision according to the actual situation.

Considering the comparison with the existing balance A, the third scheme (No. 3) in Table 5. is selected as an input to the detailed design for further optimization.

There are 12 design variables that significantly affect the performance of the sting balance, including dimensions of 2 rectangle beams and 2 horizontal $\pi$-shaped beams depicted in Fig.8. and Table 6. The other dimensions are set to constant or not involved in ADP. In ADP optimization, values of the lower bound, initial value, upper bound and increment of these variables are set through GUI of ABDOS (Table 6.), and only one objective function of stiffness is fixed. The optimum values are shown in the last column of Table 6., which are used as the initial value in FEDP optimization.
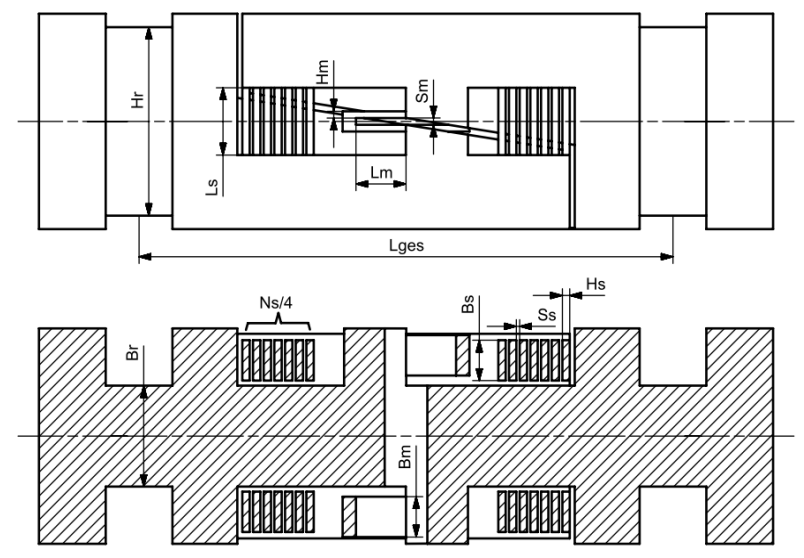

Fig.8. Geometry and parameterization of balance B for ADP.

The above optimum values of the design variables obtained in ADP optimization are imported to FEDP application as initial value with narrowing their boundaries properly to save time. New variables should be chosen for FEM simulations such as chamfers, strain gauge location, central slot angle, etc. shown in Fig.9. and Table 7., respectively. Other geometrical parameters that are predefined through GUI of ABDOS are fixed. These variables can be selected and set by the user with great flexibility. Minimizing the interference of axial component is set as another objective function which is dedicated to sting balance optimization.

Table 6. Design variables and optimum values (ADP).

\begin{tabular}{|c|c|c|c|c|c|}
\hline $\begin{array}{c}\text { Design } \\
\text { Variables } \\
(\mathrm{mm})\end{array}$ & $\begin{array}{c}\text { Lower } \\
\text { bound }\end{array}$ & $\begin{array}{c}\text { Initial } \\
\text { value }\end{array}$ & $\begin{array}{c}\text { Upper } \\
\text { bound }\end{array}$ & Increment & Optimum \\
\hline $\mathrm{Lges}$ & 140 & 150 & 170 & 1 & 155 \\
\hline $\mathrm{Br}$ & 20 & 40 & 50 & 1 & 30 \\
\hline $\mathrm{Hr}$ & 30 & 40 & 60 & 1 & 56 \\
\hline $\mathrm{Ls}$ & 8 & 10 & 25 & 1 & 20 \\
\hline $\mathrm{Bs}$ & 8 & 10 & 16 & 1 & 12 \\
\hline $\mathrm{Hs}$ & 1 & 2 & 3 & 0.1 & 2.2 \\
\hline $\mathrm{Ss}$ & 0.8 & 1 & 1.2 & 0.1 & 1 \\
\hline $\mathrm{Ns}$ & 12 & 20 & 32 & 4 & 28 \\
\hline $\mathrm{Lm}$ & 8 & 10 & 20 & 1 & 15 \\
\hline $\mathrm{Bm}$ & 8 & 10 & 16 & 1 & 12 \\
\hline $\mathrm{Hm}$ & 1 & 1.5 & 4 & 0.5 & 2 \\
\hline $\mathrm{Sm}$ & 1 & 1 & 3 & 0.5 & 2 \\
\hline
\end{tabular}

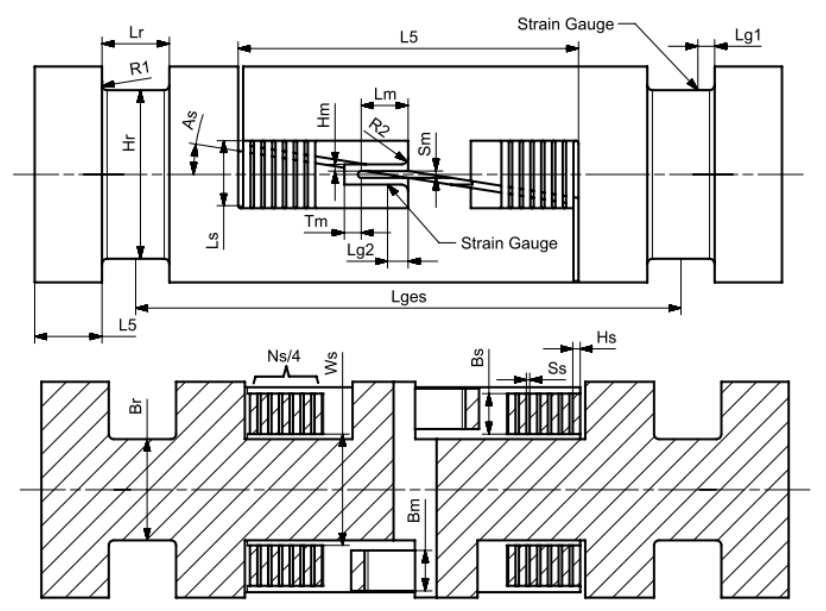

Fig.9. Geometry and parameterization of balance B for FEDP. 
Table 7. Design variables and optimum values (FEDP).

\begin{tabular}{|c|c|c|c|c|c|}
\hline $\begin{array}{c}\text { Design } \\
\text { Variables } \\
(\mathrm{mm})\end{array}$ & $\begin{array}{c}\text { Lower } \\
\text { bound }\end{array}$ & $\begin{array}{c}\text { Initial } \\
\text { value }\end{array}$ & $\begin{array}{c}\text { Upper } \\
\text { bound }\end{array}$ & Increment & Optimum \\
\hline Lges & 150 & 155 & 165 & 1 & 162 \\
\hline $\mathrm{Br}$ & 26 & 30 & 34 & 1 & 30 \\
\hline $\mathrm{Hr}$ & 48 & 56 & 58 & 1 & 50 \\
\hline $\mathrm{Ls}$ & 18 & 20 & 23 & 1 & 21 \\
\hline $\mathrm{Bs}$ & 10 & 12 & 14 & 1 & 12 \\
\hline $\mathrm{Hs}$ & 1.8 & 2.2 & 2.5 & 0.1 & 2.1 \\
\hline $\mathrm{Ss}$ & 0.8 & 1 & 1.2 & 0.1 & 1 \\
\hline $\mathrm{Ns}$ & 24 & 28 & 32 & 4 & 28 \\
\hline $\mathrm{Lm}$ & 10 & 15 & 20 & 1 & 18 \\
\hline $\mathrm{Bm}$ & 8 & 12 & 16 & 1 & 14 \\
\hline $\mathrm{Hm}$ & 1 & 2 & 4 & 0.5 & 2.5 \\
\hline $\mathrm{Sm}$ & 1 & 2 & 4 & 0.5 & 2.5 \\
\hline $\mathrm{L} 4$ & 18 & 20 & 22 & 1 & 20 \\
\hline $\mathrm{L} 5$ & 95 & 100 & 110 & 1 & 100 \\
\hline $\mathrm{Lr}$ & 18 & 19 & 22 & 1 & 20 \\
\hline $\mathrm{Lg} 1$ & 4 & 5 & 8 & 1 & 6 \\
\hline $\mathrm{Lg} 2$ & 3 & 6 & 10 & 1 & 5 \\
\hline $\mathrm{R} 1$ & 0.5 & 1 & 2 & 0.5 & 1.5 \\
\hline $\mathrm{R} 2$ & 0.5 & 1 & 2.5 & 0.5 & 2 \\
\hline $\mathrm{Tm}$ & 7 & 9 & 10 & 1 & 8 \\
\hline $\mathrm{Ws}$ & 25 & 30 & 36 & 1 & 33 \\
\hline $\mathrm{As}\left({ }^{\circ}\right)$ & 6 & 10 & 15 & 1 & 9 \\
\hline
\end{tabular}

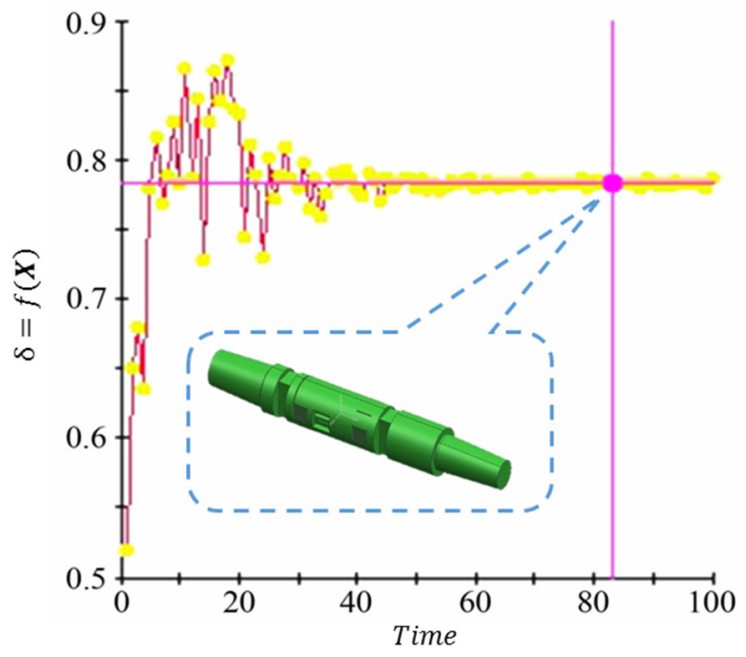

Fig.10. Iteration history of the stiffness objective function.

The final result is optimized after the evaluations of 101 schemes which are shown in the last column in Table 7 . The convergence of the deformation objective function is shown in Fig.10. The results in Table 8. show the comparison of the sensitivities of each component between balance $\mathrm{A}$ and $\mathrm{B}$ (Fig.11.). It is clear that the sensitivities of balance B are more reasonable since there is some improvement of axial component $\left(\mathrm{S}_{1}\right.$ is increased by $\left.48.1 \%\right)$, and the excessively high sensitivity components are reduced (e.g., $\mathrm{S}_{2}$ is reduced by $46.1 \%$ ). However, the rolling moment has no possibility of further improvement but it is acceptable in a sting balance. Comparing the results of the ANSYS process with the results of the Excel process, it can be found that there is a deviation in the sensitivity calculation. This is because the FEM results take into account the gradient for the stress difference between the upper and lower surfaces of the rectangular beams.

Table 9. shows the FEM simulation comparison results of the axial component interference, maximum stress and maximum deformation between balance $\mathrm{A}$ and $\mathrm{B}$. The interference by normal force on axial component is reduced from $29 \%$ to $3.5 \%$, which greatly improves the measurement accuracy of axial force. There were no significant improvements in stress and deformation $(6.7 \%$ and $4.7 \%$, respectively), which demonstrates that balance A also has a high level of quality.

Table 8. Sensitivity results and comparison.

\begin{tabular}{|c|c|c|c|c|c|c|c|}
\hline \multicolumn{2}{|c|}{$S(\mathrm{mV} / \mathrm{V})$} & $S_{1}$ & $S_{2}$ & $S_{3}$ & $S_{4}$ & $S_{5}$ & $S_{6}$ \\
\hline \multicolumn{2}{|c|}{ Balance A } & 0.52 & 2.30 & 1.25 & 0.46 & 1.52 & 2.05 \\
\hline \multirow{2}{*}{$\underset{\circlearrowright}{\tilde{\Xi}}$} & $\begin{array}{c}\text { Constrain } \\
S_{\min }\end{array}$ & 0.50 & 0.50 & 0.50 & 0.50 & 0.50 & 0.50 \\
\cline { 2 - 8 } & $\begin{array}{c}\text { Constrain } \\
S_{\max }\end{array}$ & 1.00 & 1.50 & 1.50 & 1.50 & 1.50 & 1.50 \\
\cline { 2 - 8 } & Target & 0.80 & 1.00 & 1.00 & 1.00 & 1.00 & 1.00 \\
\cline { 2 - 8 } & $\mathrm{ADP}$ & 0.71 & 0.87 & 0.65 & 0.55 & 0.93 & 0.93 \\
\cline { 2 - 8 } & FEDP & 0.77 & 1.24 & 0.89 & 0.57 & 1.05 & 1.17 \\
\hline \multicolumn{2}{|l|}{ Difference (\%) } & 48.1 & -46.1 & -28.8 & 23.9 & -30.9 & -42.9 \\
\hline
\end{tabular}

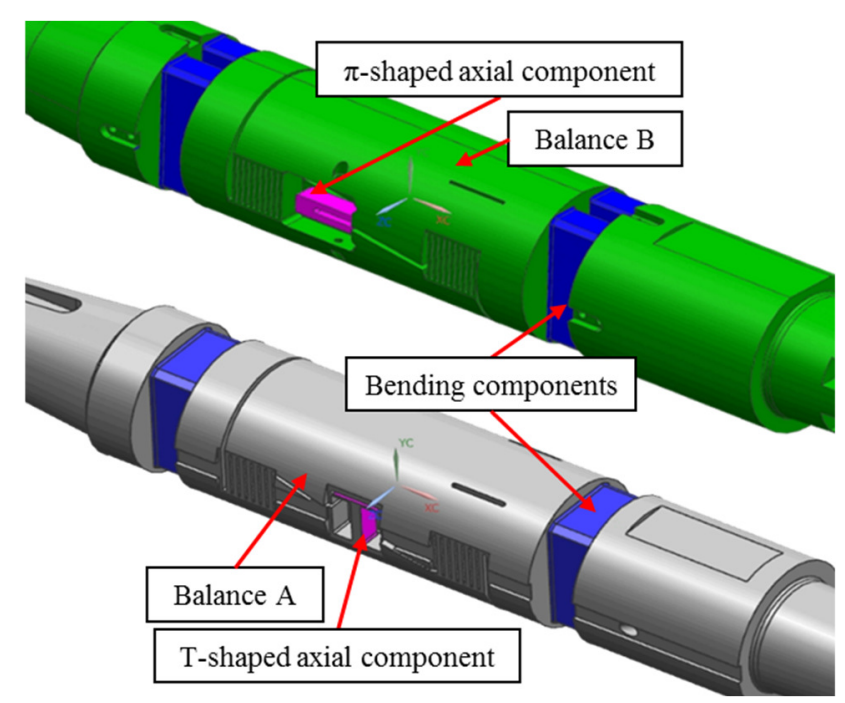

Fig.11. Structures of balance A and balance B.

The comparison of the above balance design example and results clearly shows that with the help of the new balance design optimization system, the design and optimization become easier with high efficiency and quality. Without this system, it is difficult for balance designers to find the optimal balance structure from thousands of designs in a short time. 
Table 9. Interaction, stress and deformation comparison of balance $\mathrm{A}$ and $\mathrm{B}$.

\begin{tabular}{|c|c|c|c|c|c|c|c|}
\hline & \multicolumn{5}{|c|}{$\begin{array}{c}\text { Interactions on axial component } \\
(\%)\end{array}$} & \multirow{2}{*}{$\begin{array}{l}\text { Max } \sigma \\
(\mathrm{MPa})\end{array}$} & \multirow{2}{*}{$\begin{array}{c}\operatorname{Max} \delta \\
(\mathrm{mm})\end{array}$} \\
\hline & $F_{Y}$ & $F_{Z}$ & $M_{X}$ & $M_{Y}$ & $M_{Z}$ & & \\
\hline $\mathrm{A}$ & 29 & 4.9 & 2.3 & 4.2 & 2 & 969 & 2.1 \\
\hline $\mathrm{B}$ & 3.5 & 1.6 & 1.3 & 2.7 & 1.5 & 904 & 2.0 \\
\hline
\end{tabular}

\section{CONCLUSIONS}

This paper discusses the automatic strain gauge balance design optimization method and its implementation based on the seamless integration of common application programming interface and programming languages by using both analytical methods and FEM simulation. Combined with the traditional balance design optimization idea, the mathematical modeling and strategies of the optimization system are proposed. The loop of design-simulation-redesign in the process of searching for the optimum structure and dimensions is performed automatically without the interaction of designers. The design system also widens the application of CAD-CAE software in the strain gauge balance development, further integrating the design resources and promoting the balance design technology.

The strain balance design optimization process is standardized by automating analytical and FEM simulation optimizations of the balance exploiting the iSIGHT software with integrated design experience and knowledge. Moreover, the design resources are shared and customized through the EKP remote management application. For some cases, the optimization problem could be expanded to multi-objective optimization problems without any increase in the computing cost. This black-box design approach is an implicit design mode which makes designers concentrate on the relationship between the input and output without complex design technics.

Finally, any design platform is just a tool for completing practical work and cannot absolutely replace designers at all. The integrated balance design platform only saves more time and assists designers in making better design decisions. Therefore, the balance design system does not directly help designers to carry out structural innovation, nor can it deeply understand the customer's requirement and produce a balance structure suitable for a specific project itself. At present, this integrated software system has been successfully used in the development of many conventional strain balances for wind tunnel tests. Meanwhile, it is still in the process of continuous improvement and possibly to expand to new structures and some dedicated balances in further research.

\section{REFERENCES}

[1] Tropea, C., Yarin, A.L., Foss, J.F. (2007). Springer Handbook of Experimental Fluid Mechanics. Springer, doi: 10.1007/978-3-540-30299-5.

[2] Chanetz, B. (2017). A century of wind tunnels since Eiffel. Comptes Rendus Mécanique, 345 (8), 581-594. doi:10.1016/j.crme.2017.05.012.
[3] Gebbink, R., Wang, G., Zhong, M. (2018). High-speed wind tunnel test of the CAE aerodynamic validation model. Chinese Journal of Aeronautics, 31, 439-447. doi: 10.1016/j.cja.2018.01.010.

[4] Giappino, S., Melzi, S., Tomasini, G. (2018). Highspeed freight trains for intermodal transportation: Wind tunnel study on the aerodynamic coefficients of container wagons. Journal of Wind Engineering and Industrial Aerodynamics, 175, 111-119. doi: 10.1016/ j.jweia.2018.01.047.

[5] Sheng, R., Perret, L., Calmet, I., Demouge, F., Guilhot, J. (2018). Wind tunnel study of wind effects on a highrise building at a scale of 1:300. Journal of Wind Engineering and Industrial Aerodynamics, 174, 391403. doi:10.1016/j.jweia.2018.01.017.

[6] Gorlin, S.M., Slezinger, I.I. (1964). Wind Tunnels and Their Instrumentation. IPST, 599.

[7] Damljanović, D., Isaković, J., Rašuo, B. (2013). T-38 wind-tunnel data quality assurance based on testing of a standard model. Journal of Aircraft, 50 (4), 11411149. doi: 10.2514/1.c032081.

[8] Vidanović, N.D., Rašuo, B.P., Damljanović, D.B., Vuković, D.S., Curčić, D.S. (2014). Validation of the CFD code used for determination of aerodynamic characteristics of nonstandard AGARD-B calibration model. Thermal Science, 2014, 18 (4), 1223-1233. doi:10.2298/TSCI130409104V.

[9] Ocokoljić, G., Damljanović, D., Vuković, Đ., Rašuo, B.P. (2018). Contemporary frame of measurement and assessment of wind-tunnel flow quality in a low-speed facility. FME Transactions, 46 (4), 429-442. doi: 10.5937/fmet18044290.

[10] Ewald, B.F.R. (2000). Multi-component force balances for conventional and cryogenic wind tunnels. Measurement Science and Technology, 11 (6). doi: 10.1088/0957-0233/11/6/201.

[11] Parker, P.A. (2001). Cryogenic balance technology at the National Transonic Facility. In 39th AIAA Aerospace Sciences Meeting \& Exhibit. AIAA-20010758.

[12] Burns, D.E., Williams, Q.L., Phillips, B.D., Commo, S.A. (2016). Review of potential wind tunnel balance technologies. In $10^{\text {th }}$ International Symposium on Strain-Gauge Balances.

[13] Hou, J.W., Twu, S.L. (1987). Optimum design of internal strain-gage balances: An example of threedimensional shape optimization. Journal of Mechanical Design, 109 (2), 257-262. doi:10.1115/1.3267448.

[14] Lindell, M.C., Center, L.R. (1996). Finite element analysis of a NASA National Transonic Facility wind tunnel balance. In International Symposium on StrainGauge Balances.

[15] Zhai, J., Ewald, B., Hufnagel, K. (1995). Investigation on the interference of internal six-component wind tunnel balances with FEM. In ICIASF'95 Record. International Congress on Instrumentation in Aerospace Simulation Facilities. IEEE. 
[16] Parker, P.A., DeLoach, R. (2002). Structural optimization of a force balance using a computational experiment design. In 40th AIAA Aerospace Sciences Meeting \& Exhibit, AIAA 2002-0540.

[17] Rhew, R.D. (2005). Strain-Gage balance axial section design optimization using design of experiments. In U.S. Air Force T\&E Days, AIAA 2005-7600. doi: 10.2514/6.2005-7600.

[18] Sun, Y., Liu, Y., Zou, T., Jin, M., Liu, H. (2015). Design and optimization of a novel six-axis force/torque sensor for space robot. Measurement, 65, 135-148. doi: 10.1016/j.measurement.2015.01.005.

[19] Vadassery, P., Joshi, D.D., Rolim, T.C., Lu, F.K. (2013). Design and testing of an external drag balance for a hypersonic shock tunnel. Measurement, 46, 2110 2117. doi: 10.1016/j.measurement.2013.03.011.

[20] Nouri, N.M., Mostafapour, K., Kamran, M., Bohadori, R. (2014). Design methodology of a six-component balance for measuring forces and moments in water tunnel tests. Measurement, 58, 544-555. doi: 10.1016/j.measurement.2014.09.011.

[21] Lynn, K.C. (2015). Flexural fillet geometry optimization for design of force transducers used in aerodynamic testing. In 53rd AIAA Aerospace Sciences Meeting, AIAA 2015-1789. doi: 10.2514/6.2015-1789.

[22] Tavakolpour-Saleh, A.R., Setoodeh, A.R., Gholamzadeh, M. (2016). A novel multi-component strain-gauge external balance for wind tunnel tests: Simulation and experiment. Sensors and Actuators, A: Physical, 247, 172-186. doi:10.1016/j.sna.2016.05.035.
[23] Kolhapure, R., Shinde, V., Kamble, V. (2017). Geometrical optimization of strain gauge force transducer using GRA method. Measurement, 101, 111117. doi: 10.1016/j.measurement.2017.01.030.

[24] Akbari, H., Kazerooni, A. (2018). Improving the coupling errors of a Maltese cross-beams type six-axis force/moment sensor using numerical shapeoptimization technique. Measurement, 126, 342-355. doi: 10.1016/j.measurement.2018.05.074.

[25] Park, H.S., Dang, X.P. (2010). Structural optimization based on CADCAE integration and metamodeling techniques. Computer-Aided Design, 42 (10), 889-902. doi: 10.1016/j.cad.2010.06.003.

[26] Chandrasegaran, S.K., Ramani, K., Sriram, R.D., Horváth, I., Bernard, A., Harik, R.F., Gao, W. (2013). The evolution, challenges, and future of knowledge representation in product design systems. ComputerAided Design, 45 (2), 204-228. doi: 10.1016/j.cad. 2012.08.006.

[27] Dang, X.P. (2014). General frameworks for optimization of plastic injection molding process parameters. Simulation Modelling Practice and Theory, 41, 15-27. doi: 10.1016/j.simpat.2013.11.003.

[28] Vidanović, N., Rašuo, B., Kastratović, G., Maksimović, S., Ćurčić, D., Samardžić, M. (2017). Aerodynamicstructural missile fin optimization. Aerospace Science and Technology, 65, 26-45. doi: 10.1016/j.ast.2017.02. 010.

[29] Dassault Systèmes SIMULIA. (2012). Isight 5.7. User's Guide. 